\title{
The Geometry of Behavioral and Brain Dynamics in Team Coordination
}

\author{
Silke Dodel ${ }^{1}$, Emmanuelle Tognoli ${ }^{1}$, and J.A. Scott Kelso ${ }^{1,2}$ \\ 1 Center for Complex Systems and Brain Sciences, Florida Atlantic University, \\ Boca Raton, FL 33431, USA \\ 2 Intelligent Systems Research Center, University of Ulster, Derry, N. Ireland \\ dodel@ccs.fau.edu
}

\begin{abstract}
Performing a task as a team requires that team members mutually coordinate their actions. It is this coordination that distinguishes the performance of a team from the same actions performed independently. Here we set out to identify signatures of team coordination in behavioral and brain dynamics. We use dual electroencephalography (EEG) to measure brain dynamics of dyadic teams performing a virtual room clearing task. Such complex tasks often exhibit high variability of behavioral and brain dynamics. Although such variability is often considered to impede identification of the behavior or brain dynamics of interest here we present a conceptual and empirical framework which explains variability in geometrical terms and classifies its sources into those that are detrimental and non-detrimental to performing the task at hand. Using our framework we found that behaviorally team coordination is reflected in terms of role dependent behavior. Furthermore we identified a low-dimensional subspace of the brain dynamics in the frequency domain which is specific for team behavior and correlated with successful team coordination. Moreover, successful team coordination was positively correlated with the inter- but not intra-brain coherence in the gamma band. Our results hence indicate that successful team coordination is associated with increased team cognition, particularly readiness to engage in the task.
\end{abstract}

\section{Introduction}

Many tasks in real life are best accomplished when a group of people acts as a team. Examples include lifting heavy weights, hunting, police or military operations, and team sports, but also some instances of abstract problem solving. Performing a task as a team requires that team members mutually coordinate their actions. It is this coordination that distinguishes the performance of a team from the same actions performed independently by multiple subjects. While multiple studies investigate team cognition from behavioral measures [12345678910, it is currently unknown how team cognition is reflected in the brain dynamics of the team members. Studies of brain activity during social interaction - but not team cognition - have found brain rhythms associated with social coordination [1], inter-brain coherence 12 13, inter-brain 
Granger causality [14, and joint brain networks [15]. The findings of the few extant studies about brain activity during team tasks include indications of interbrain functional connectivity in partners of the same team in a card game [16], dimensionality of brain dynamics being affected by team expertise and task difficulty [17, and classification of EEG engagement patterns of individual team members [18.

Here we use dual electroencephalography (EEG) of dyadic teams who perform a virtual room clearing task to identify neuromarkers of team coordination. The task we use is a virtualization of one of the most extreme forms of team coordination, namely when members' survival and safety depend upon efficient team interactions. During such tasks cognitive and social processes have to be coordinated in a context-dependent fashion. The task is ecologically valid and highly dynamic with well defined behavior in which subjects dynamically engage in and disengage from team coordination. Team coordination can be studied in a meaningful way only in ecologically valid tasks. Ecologically valid tasks however are often highly dynamic and individuals performing such tasks exhibit a high variability of behavioral and brain dynamics. Here we present a conceptual framework which explains this variability in geometrical terms and classifies variability into detrimental and non-detrimental to the task at hand. Our framework provides a unifying theoretical account for tasks with multiple degrees of freedom and hence is particularly suited to guide the analysis of team tasks.

The paper is organized as follows: In section 2.1 we describe the experiment, in section 2.2 the conceptual framework for analyzing behavioral and brain dynamics in complex tasks is introduced, and in section 3 we present the results of this framework, followed by a discussion in section 4 .

\section{Materials and Methods}

\subsection{Virtual Room Clearing Task}

For our study we devised a virtual room clearing task by creating a video game in which team members of a dyadic team work together to detect and eliminate enemies as they jointly progress through buildings in a hostile urban environment, with the shared goal of clearing a virtual room from threats. The video game was designed to retain the essence of key behavioral, perceptual, cognitive, social and attentional processes that occur in successful team work. Subjects shared the same top down perspective of their virtual environment while controlling their avatar's position and direction of gaze, as they navigated through a series of 32 buildings each composed of 5 successive rooms, with their virtual environment becoming visible upon the avatars' spatial exploration. Dual-EEG was recorded by using two 60 channel EEG caps and a sampling rate of $1 \mathrm{kHz}$. The experimental setup is described in more detail in [19]. Figure 1)(a) shows an example trajectory of an avatar dyad through a building. In each room clearing task one subject assumes the role of the leader and the other the role of the follower. The subjects assume the roles of leader and follower in a self-organized manner and renegotiate their roles implicitly by the behavior of their avatars 
after completion of each room clearing trial. There are two valid entry patterns, the leader button-hook entry and the leader cross-over entry (cf. Figure 1(b)). Due to this degeneracy, the task is endowed with intrinsic variability. On the day before the experiment, subjects received training for one hour to master basic concepts of room clearing. Although in our study the room clearing task is virtual, the behavior of the avatars closely resembles the behavior of subjects performing an actual room clearing task (compare Fig. 1(c) to Fig. 3, left).

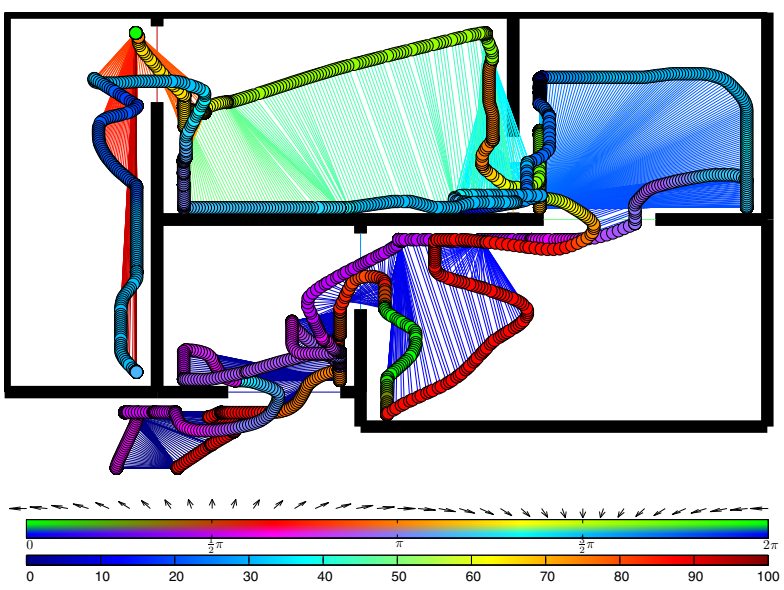

(a)
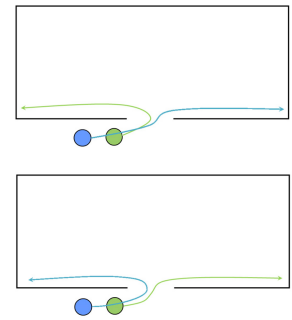

(b)

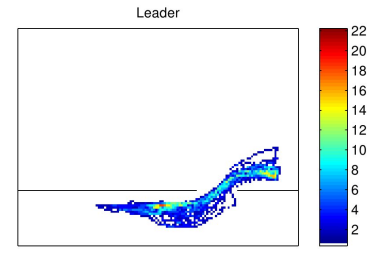

(c)

Fig. 1. (a) Trajectories of the avatar dyad through the five rooms of one of the buildings. Color of the beams between team member positions: percentage of trajectory completed (from blue $0 \%$ to red 100\%). Color of the circles: Gaze direction. Green subject: green $0^{\circ}$, red $120^{\circ}$, yellow $240^{\circ}$. Blue subject: blue $0^{\circ}$, magenta $120^{\circ}$, cyan $240^{\circ}$. (b) Two possible team entry patterns: leader button-hook (top) and leader crossover (bottom). The follower executes the complementary entry pattern, respectively. (c) Spatial probability density of leader trajectories in a behavioral (not virtual) room clearing task. Values indicate the percentage of trajectories crossing a given region. Based on data from 8 .

Each room clearing trial proceeds as follows: The follower aligns behind the leader. The follower gives a ready signal, conveyed through touch 'tap' in the real situation, and through a vibration of the Xbox controller in the experiment (verbal communication was not allowed to avoid EEG artifacts) upon which the leader initiates motion of his avatar and is followed by the avatar of the follower. Then both subjects move their avatars to the door as close as possible and the leader presses a button to open the door. The leader enters the room, followed as closely as possible by the follower. Then both leader and follower independently move to their respective corners of dominance, which are the corners to the left 
and right from the door. Both corners of dominance must be covered, hence the follower needs to coordinate his entry pattern with that of the leader such as to move to the opposite corner as the leader. After arriving in their corners of dominance, subjects had instruction to engage in gaze interlock, i.e. the overlap of their view cones. The gaze interlock ended the trial and the subjects moved to the next stacking point. From the description above, each room clearing trial can be divided into three main events: First, the coordination build-up while stacking in front of the room, second, coordinated behavior when moving to the door and entering the room, and third, breaking and rebuilding coordinated behavior.

\subsection{Geometrical Description of Behavioral and Brain Dynamics during Complex Tasks}

Detrimental and Non-detrimental Variability. Ecologically valid tasks are often complex and have a high number of degrees of freedom which leads to behavioral variability because there are multiple valid ways in which these tasks can be executed (cf. Fig. 1(b)). Such behavioral variability is non-detrimental to task execution and actually may improve its performance metrics (e.g. speed, survival, ...). However, other variability may be detrimental, representing a deviation from ideal task execution. Furthermore in team tasks the behavior of each team member is influenced by the behavior of the other team members. This gives rise to particular behavioral patterns which are not present in tasks performed by a single subject. In addition to behavioral variability there is also variability in the brain dynamics associated with task execution. Neural processes also exhibit degeneracy, and hence a single behavior may be supported by multiple neural processes.

To guide the choice and development of analysis methods we devised a geometrical framework for the description of complex tasks. This allows to translate properties of the behavioral and brain dynamics into geometrical properties and makes available geometrical tools for the analysis of dynamics in brain and behavior. An implicit geometrical perspective is already adopted in many standard multivariate analysis methods such as principal component analysis or clustering algorithms. Our framework is novel however, because it offers a conceptualization of all behavioral aspects of a task and its associated brain dynamics in terms of geometry and furthermore provides a geometrical account for inter subject coordination.

Behavioral and Brain Manifolds. Behavioral and brain dynamics during a complex task can be described geometrically as a trajectory in a phase space. Each dimension of the phase space represents a variable of the dynamics. The choice of the phase space is not unique. Different phase spaces hence provide different "windows" to the problem. The brain dynamics in each trial represents a trajectory in phase space. The ensemble of all possible trajectories constitutes a geometrical object which we refer to as manifold. The term "manifold" is chosen 
to indicate that the geometrical object can have a non-trivial shape and alludes to the Uncontrolled Manifold in movement sciences [20. Here we extend the uncontrolled manifold concept to team behavior and brain dynamics. Figure 2 shows the manifold concept for behavioral and brain dynamics, respectively. A trajectory on the behavioral manifold contains information about the team behavior in a given trial, and a trajectory in the brain manifold represents information about the associated brain dynamics. Note that unless stated otherwise, the term "trajectory" always refers to a trajectory on the manifold rather than to a physical trajectory through the virtual rooms. The shape of the manifolds is governed by three main factors: task constraints, team coordination and task performance. Task constraints affect the global shape of the manifold while team coordination and task performance delineate submanifolds.

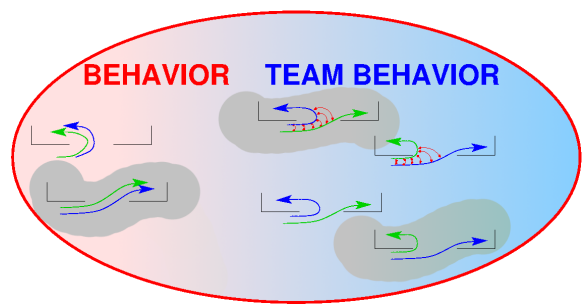

(a) Behavioral manifold

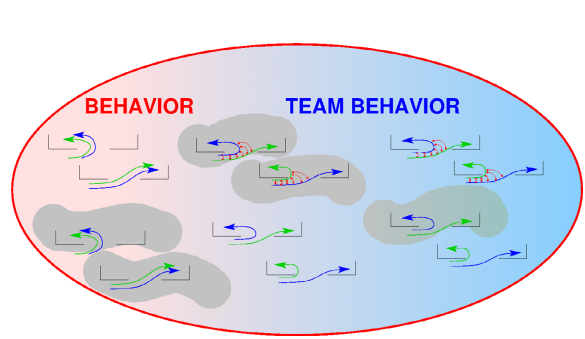

(b) Brain manifold (team coordination: modulation of neural processes)

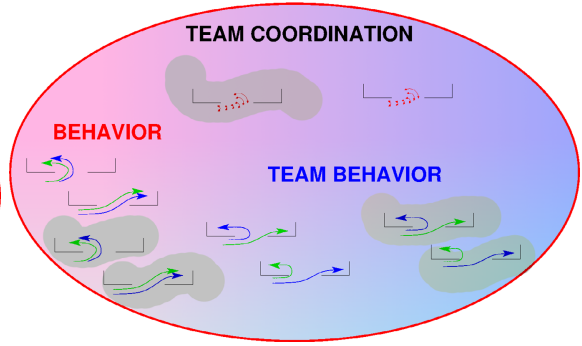

(c) Brain manifold (team coordination: separate neural processes)

Fig. 2. Illustration of the manifold concept in phase spaces of behavioral and brain dynamics, respectively. (a) Behavioral manifold. (b) Brain manifold in which team coordination is supported by modulation of neural processes underlying behavior. (c) Brain manifold in which coordination is supported by separate neural processes. The cartoons on the brain manifold stand for the behavior that the neural processes support. Red connections illustrate team coordination. Due to degeneracy, the same behavior may be supported by different neural processes. In all manifolds, team performance increases from left to right, and team coordination from bottom to top. The shaded areas in the manifolds illustrate a probability measure on the manifold.

Team Subspace. A manifold can be approximated at any given point by its tangent space at this point. Here we use singular value decomposition (SVD) of 
local time-frequency windows to approximate the tangent spaces of the manifolds. Neuromarkers are properties of brain dynamics that are related to a certain behavior, which in our case is team coordination. For neuromarkers to be identifiable, certain properties of brain dynamics during the behavior in question need to be sufficiently consistent. Consistency, however, implies low variability and hence we are confronted with the challenge to identify consistent patterns from highly variable brain dynamics. To identify consistent brain dynamics related to team behavior, yet account for the variability associated with neural and behavioral degeneracy, we compute the intersection of the tangent spaces over different trials and project the brain signals into this subspace which we refer to as team subspace. The subspace represents the submanifold of the brain manifold which is related to team behavior and the projected brain signals correspond to trajectories on this submanifold. We determined the intersection of the tangent spaces by computing the principal angles between them using a method proposed in [17. The dimensionality of the team subspace was determined by the number of dimensions for which $\cos \alpha>0.8$ where $\alpha$ is a principal angle.

Measure of Team Coordination. When the subjects perform the virtual room clearing task, task initiation is a key event of team coordination. The task is initiated when the follower taps the leader to signal his readiness to start upon which the leader starts to move. A short duration of this time interval indicates that both subjects were simultaneously ready to start the task. Here we use the duration of task initiation as global measure of team coordination of a trial. Short durations thereby indicate successful team coordination.

\section{Results}

Behavioral Signatures of Team Coordination. Our study focusses on brain signatures of team coordination, however, we found that behavioral signatures of team coordination were present even on the level of single subject behavior. More specifically, we found that the entry patterns of leaders and followers exhibited role-dependent differences. Figure 3 shows the behavioral variability over trials of cross-over entries performed by a leader and a follower, respectively. The entry pattern of the leader has much less variability than that of the follower. There are two regions of high concentration of leader entry trajectories, one before and one after the entry. Interestingly, this pattern is also found in the leader trajectory during actual room clearing (cf. Fig. 1(c)). The entry pattern of the follower is much more diffuse with only one area of slightly higher concentration of entry trajectories. This indicates that the follower is coordinating with the leader. The coordination of the follower with the leader has the effect of enhancing the variability of his own behavior, since his behavior is affected by both external task constraints and the behavior of the leader, while the leader's behavior is primarily affected by external task constraints. 

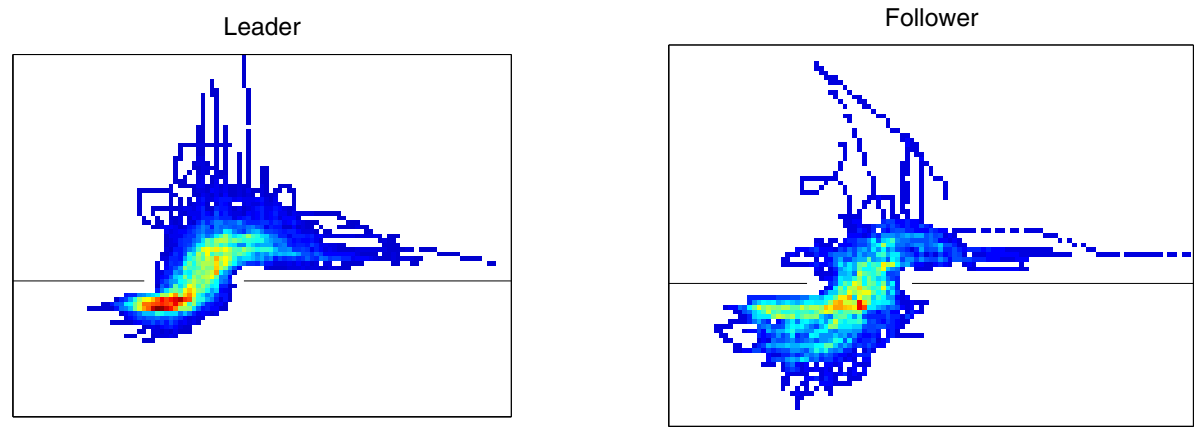

$2 \%$

(a)

(b)

Fig. 3. Behavioral variability of a cross-over entry of a leader and a follower. Spatial probability density of the trajectories. Values indicate the percentage of trajectories crossing a given region. (a) Leader. (b) Follower.

Brain Signatures of Team Coordination. We applied our geometrical framework (cf. Materials and Methods) to the dual EEG data from the virtual room clearing task and determined a brain manifold in the frequency domain, using as phase space variables the real and imaginary parts of a wavelet transform (complex Morlet, 3 peaks) of the signal of all electrodes. We restricted our analysis to the frequency band of $15-40 \mathrm{~Hz}$ ( $\beta$ and low and medium $\gamma$ band), because the behaviors of interest like signaling readiness by the follower, movement initiation by the leader, and entry coordination between the two subjects occur at time scales of $200 \mathrm{~ms}$ or less, corresponding to 3-25 cycles in the chosen frequency band. Here we report results from the task initiation interval, which is the interval between the tap performed by the follower to signal readiness and the movement onset of the leader. This interval has a duration of $250 \mathrm{~ms}-$ $1000 \mathrm{~ms}$ with an average around $400 \mathrm{~ms}$. Figure 4(a) shows the spatio-temporal patterns of the wavelet powers in the frequencies 15-40 Hz during the first 250 ms of the task initiation interval, averaged over trials for one dyadic team. We found consistent low-dimensional team subspaces (cf. Materials and Methods) for each dyadic team, with dimensionalities of 8-11. Neuromarkers of team behavior were identified by the portion of the brain signals that lay within the team subspace (cf. Fig. 4(b)). We found the mean relative wavelet power in the team subspaces to be significantly positively correlated with successful team coordination as measured by the duration of the task initiation interval (cf. Materials and Methods). This supports the notion of a low-dimensional subspace of joint brain activity which is related to team coordination. Moreover we found that interbrain coherence, but not intra-brain coherence in the low and medium $\gamma$ bands $(30-40 \mathrm{~Hz})$ during task initiation was significantly positively correlated with 
(a) Wavelet powers
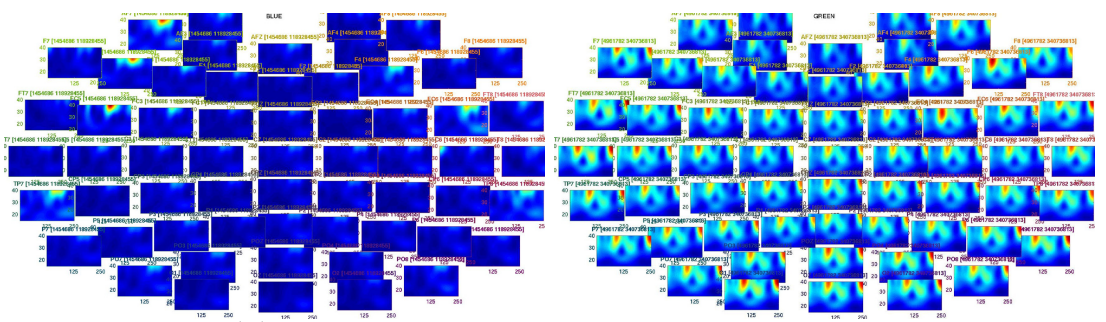

(b) Wavelet powers within the team subspace
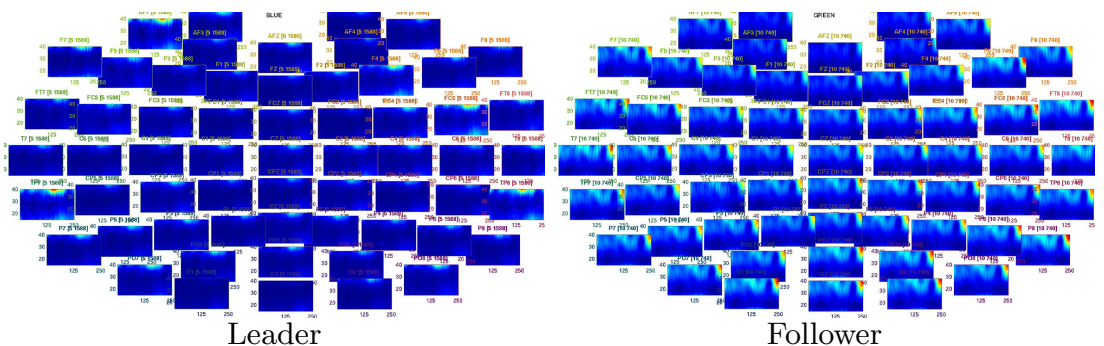

Fig. 4. Mean wavelet powers of leader (left column) and follower (right column) (a) before and (b) after projection onto the team subspace

successful team coordination as well. With inter-brain coherence and spatiotemporal wavelet powers in a team subspace, we have identified two neuromarkers of team coordination.

\section{Discussion}

Social interaction is a crucial part of human life. Yet the neuroscience of social interaction is only in its infancy. To identify neuromarkers of team coordination we have performed a dual EEG study of dyadic teams performing a virtual room clearing task. Ecologically valid tasks like this represent a particular challenge to the analysis because of their high inherent variability. We have developed a geometrical framework that provides a unifying theoretical account for ecologically valid tasks and is particularly suited to guide the analysis of team tasks. In this framework behavioral and brain dynamics are interpreted as evolving along trajectories on a manifold in a high-dimensional phase space. The geometry of the manifold is determined by multiple factors, such as task constraints, team performance and team coordination. The manifold depends on the choice of the phase space. Here we analyzed the data in the wavelet domain, which lends itself to two natural descriptors: wavelet power and coherence, representing amplitude and phase information, respectively.

While our focus was on neuromarkers, we found behavioral signatures of team coordination even on the single subject level in terms of role dependent behavior. 
Their pattern indicates that the follower is coordinating his behavior with that of the leader akin to an object attached to the leader. On the level of the brain, successful team coordination was associated with an increased proportion of the subjects' brain signals evolving in a low-dimensional team subspace. Moreover, we found successful team coordination to be positively correlated with interbut not intra-brain coherence in the $\gamma$ band during task initiation. This implies that inter-brain coherence is higher when both subjects are ready to engage in the task. Since task initiation is associated with information transfer, inter-brain coherence might be a signature of a state of mind of both subjects which enables or facilitates information transfer. While it has been argued that coherently oscillating neuronal groups facilitate efficient interaction within a brain [21, there is currently no extablished model of how inter-brain coherence could be mediated [22. A possible interpretation of our finding is that inter-brain coherence is related to the subjects' receptiveness of their mutual behavior. However, a more detailed analysis of the spatio-temporal patterns of intra- and inter-brain coherence in relation to the subjects' behavior is needed to test this hypothesis.

Behavioral variability is brought about by the degrees of freedom of the task as well as differences in task performance. Variability in brain dynamics is due to behavioral variability and due to the degeneracy of brain processes. It is well known - although rarely explicitly acknowledged - that multiple different brain processes may support identical behavior [23. Our framework represents a departure from the quest for single neural mechanisms underlying given behaviors and explicitly acknowledges the degeneracy and highly synergistic nature of brain processes.

\section{References}

1. Fiore, S.M., Salas, E., Cuevas, H.M., Bowers, C.A.: Distributed coordination space: Toward a theory of distributed team process and performance. Theoretical Issues in Ergonomics Science 4(3-4), 340-364 (2003)

2. Cooke, N.J., Gorman, J.C., Duran, J.L., Taylor, A.R.: Team cognition in experienced command-and-control teams. J. Exp. Psychol. Appl. 13(3), 146-157 (2007)

3. Woolley, A.W., Hackman, R.J., Jerde, T.E., Chabris, C.F., Bennett, S.L., Ko sslyn, S.M.: Using brain-based measures to compose teams: How individual capabilities and team collaboration strategies jointly shape perf ormance. Social Neuroscience 2(2), 96-105 (2007)

4. Salas, E., Cooke, N.J., Rosen, M.A.: On teams, teamwork, and team performance: discoveries and developments. Hum Factors 50(3), 540-547 (2008)

5. Bourbousson, J., Sve, C., McGarry, T.: Space-time coordination dynamics in basketball: Part 1. intra- and inter-couplings among player dyads. J. Sports Sci. 28(3), 339-347 (2010)

6. Bourbousson, J., Sve, C., McGarry, T.: Space-time coordination dynamics in basketball: Part 2. the interaction between the two teams. J. Sports Sci. 28(3), 349-358 (2010)

7. DeChurch, L.A., Mesmer-Magnus, J.R.: The cognitive underpinnings of effective teamwork: a meta-analysis. J. Appl. Psychol. 95(1), 32-53 (2010) 
8. Dodel, S., Pillai, A., Fink, P., Muth, E., Stripling, R., Schmorrow, D., Cohn, J., Jirsa, V.: Observer-independent dynamical measures of team coordination and performance. Motor Control: Theories, Experiments, and Applications, 72-101 (2010)

9. Gorman, J.C., Amazeen, P.G., Cooke, N.J.: Team coordination dynamics. Nonlinear Dynamics Psychol Life Sci. 14(3), 265-289 (2010)

10. Gorman, J.C., Cooke, N.J.: Changes in team cognition after a retention interval: The benefits of mixing it up. J. Exp. Psychol. Appl. 17(4), 303-319 (2011)

11. Tognoli, E., Lagarde, J., DeGuzman, G.C., Kelso, J.A.S.: The phi complex as a neuromarker of human social coordination. Proc. Natl. Acad. Sci. U S A 104(19), 8190-8195 (2007)

12. Lindenberger, U., Li, S.C., Gruber, W., Müller, V.: Brains swinging in concert: cortical phase synchronization while playing guitar. BMC Neurosci. 10, 22 (2009)

13. Dumas, G., Nadel, J., Soussignan, R., Martinerie, J., Garnero, L.: Inter-brain synchronization during social interaction. PLoS One 5(8), e12166 (2010)

14. Schippers, M.B., Roebroeck, A., Renken, R., Nanetti, L., Keysers, C.: Mapping the information flow from one brain to another during gestural communication. Proceedings of the National Academy of Sciences 107(20), 9388-9393 (2010)

15. Anders, S., Heinzle, J., Weiskopf, N., Ethofer, T., Haynes, J.D.: Flow of affective information between communicating brains. NeuroImage 54(1), 439-446 (2011)

16. Astolfi, L., Toppi, J., De Vico Fallani, F., Vecchiato, G., Salinari, S., Mattia, D., Cincotti, F., Babiloni, F.: Neuroelectrical hyperscanning measures simultaneous brain activity in humans. Brain Topography 23, 243-256 (2010), doi:10.1007/s10548-010-0147-9

17. Dodel, S., Cohn, J., Mersmann, J., Luu, P., Forsythe, C., Jirsa, V.: Brain signatures of team performance. Foundations of Augmented Cognition. Directing the Future of Adaptive Systems, 288-297 (2011)

18. Stevens, R.H., Galloway, T.L., Wang, P., Berka, C.: Cognitive neurophysiologic synchronies: What can they contribute to the study of teamwork? Human Factors: The Journal of the Human Factors and Ergonomics Society (2011)

19. Tognoli, E., Kovacs, A., Suutari, B., Afergan, D., Coyne, J., Gibson, G., Stripling, R., Kelso, J.: Behavioral and brain dynamics of team coordination part i: Task design. Foundations of Augmented Cognition. Directing the Future of Adaptive Systems, 257-264 (2011)

20. Scholz, J., Schöner, G.: The uncontrolled manifold concept: identifying control variables for a functional task. Experimental Brain Research 126(3), 289-306 (1999)

21. Bressler, S.L., Tognoli, E.: Operational principles of neurocognitive networks. International Journal of Psychophysiology 60(2), 139-148 (2006)

22. Dumas, G., Chavez, M., Nadel, J., Martinerie, J.: Anatomical connectivity influences both intra- and inter-brain synchronizations. PLoS One 7(5), e36414 (2012)

23. Price, C.J., Friston, K.J.: Degeneracy and cognitive anatomy. Trends Cogn. Sci. 6(10), 416-421 (2002)

24. De Jaegher, H., Di Paolo, E., Gallagher, S.: Can social interaction constitute social cognition? Trends Cogn. Sci. 14(10), 441-447 (2010) 